\title{
Functioning Pituitary Gland Neoplasm
}

National Cancer Institute

\section{Source}

National Cancer Institute. Functioning Pituitary Gland Neoplasm. NCI Thesaurus. Code

C7047.

A hormone producing pituitary gland tumor, associated with a hormonal syndrome. 